\title{
A Comparison of Gamma Irradiation Response Models of Bovine Blood, Liver and Kidney Tissues at Radiofrequency
}

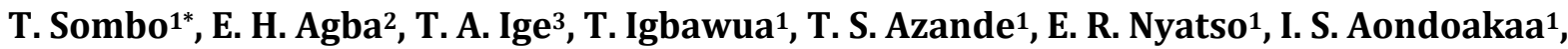 \\ T. J. Shivil ${ }^{1}$
}

${ }^{1}$ Department of Physics, Federal University of Agriculture, Makurdi, Nigeria

${ }^{2}$ Department of Physics, Benue State University, Makurdi, Nigeria

${ }^{3}$ Department of Physics, National Hospital, Abuja, Nigeria

Email: ^jtsombo@gmail.com

How to cite this paper: Sombo, T., Agba, E.H., Ige, T.A., Igbawua, T., Azande, T.S., Nyatso, E.R., Aondoakaa, I.S. and Shivil, T.J. (2018) A Comparison of Gamma Irradiation Response Models of Bovine Blood, Liver and Kidney Tissues at Radiofrequency. Open Journal of Biophysics, 8, 176-183.

https://doi.org/10.4236/ojbiphy.2018.83013

Received: December 26, 2017

Accepted: July 22, 2018

Published: July 25, 2018

Copyright $\odot 2018$ by authors and Scientific Research Publishing Inc. This work is licensed under the Creative Commons Attribution International License (CC BY 4.0).

http://creativecommons.org/licenses/by/4.0/

CC) (i) Open Access

\begin{abstract}
This study is aimed at identifying gamma irradiated response of bovine blood, liver and kidney tissues at radiofrequency. For this purpose, impedence meter (Booton 7200) working in conjunction with signal generators (Loadster, SG416013 and Harris G857993) and improvised parallel plate dielectric cells constructed in line with the method of Laogun et al., (2005) were used to obtain the dielectric spread parameter gamma radiation $\alpha$, of blood, liver and kidney tissues exposed to gamma irradiation dose range of 1.0 - 85.0 Gy. The dielectric spread parameter $\alpha$ gives the extent of damage induced in an irradiated tissue. Results of this work revealed that at $0-50 \mathrm{MHz}$ frequency range, Kidney tissues displayed higher sensitive, followed Liver tissues and lasted the bovine blood between 0 - 60 Gy but reversed for blood and liver at $85 \mathrm{~Gy}$. At $0-100 \mathrm{kHz}$ frequency range liver tissue is more venerable to radiation injuries between gamma irradiation dose range of 0 - 20 Gy while between 43 - 85 Gy the Kidney's sensitivity is the highest followed by blood and liver tissues. This implies that the liver tissues are less liable to radiation injuries at radiofrequency. A comparison of the linear, exponential and polynomial models using Akaike Information Criteria (AIC) revealed that linear models were the most suitable models for describing the effect of gamma radiation on the dielectric dispersion properties of bovine tissues at low and high radiofrequencies. This implies that the response of the investigated tissues increases linearly with gamma irradiation dose.
\end{abstract}

\section{Keywords}

Gamma Irradiation, Dielectric Spread Parameter, Mathematical Models, AIC, Bovine Tissues 


\section{Introduction}

The effect of gamma radiation on human tissues has been extensively studied and findings revealed that its beneficial effects are employed in the diagnosis and treatment of various ailments like cancers. Its non-beneficial effects include the induction of cancers, genetic disorder (mutation), and other numerous stochastic and non-stochastic effects [1]. These effects arise from the excitation, ionization and breakage of some chemical bonds in the molecules of the exposed tissue [2]. This leads to the production of complex ions (radicals) which reacts to produce toxins. These toxins are circulated throughout the body via lymphatic circulation and bloodstream causing radiation sicknesses [3].

The level of damage induced in an organ (tissue) depends on the quantity of radiation absorbed by the tissues, its composition and morphology. This implies that some tissues are more prone to radiation than others. The interaction of complex ions produced as a result of ionization event in the molecules of the irradiated tissues leads to the generation of toxins. These toxins are circulated throughout the body via lymphatic circulation and bloodstream [3]. Syndromes of Acute Radiation Sickness (ARS) are expressed depending on the type of induced toxin.

Previous studies [4] [5] [6] [7] [8] have focused attention on the dielectric characterization of tissues whereas virtually little or no information exists on development of models that explains the difference in radio sensitivity between non-lymphoid tissues (liver and kidney) and moving mammalian lymphoid tissues (blood) based on dielectric relaxation spread [9]. Hence, the need to direct efforts towards development of and comparison of response models of lymphoid and non-lymphoid tissues via mathematical models.

\section{Materials and Methods}

The liver and kidney tissue samples were excise from an adult white Fulani cow which was duly certified healthy by veterinary doctor attached to Gwagwalada Central Abattoir in Gwagwalada Area Council, Abuja, Nigeria. The excised tissue samples were thoroughly washed with double distilled water and preserved in laboratory oven maintained at a temperature of $37.0^{\circ} \mathrm{C} \pm 0.5^{\circ} \mathrm{C}$ for six hours to remove water from their surfaces. The blood samples were collected from the certified cow by vein puncture using a $10 \mathrm{ml}$ hypodermic needle attached to a disposable plastic syringe. The blood samples were immediately introduced into heparinized tubes to prevent coagulation. Percentage water content of blood, kidney and liver samples were $86.0 \%, 72.2 \%$ and $71.6 \%$ respectively.

Eight portions of each of the three tissue samples were irradiated at the following irradiation doses; 1.0 Gy, 4.0 Gy, 11.0 Gy, 20.0 Gy, 43.0 Gy, 60.0 Gy and 85.0 Gy respectively using the Gamma Irradiator (GS 1000) located at the Gamma Irradiation Facility (GIF) Unit of the National Nuclear Technology Centre, Abuja at a dose rate of $0.36 \mathrm{kGy} / \mathrm{hr}$ after which the various sample were introduced into their respective dielectric cells. The dielectric sample cells used in this 
research were constructed and calibrated in line with the method of Laogun [6] and Agba et al. [7]. The dielectric constant, $\mathrm{k}$ and residual capacitance of the dielectric cells constructed for blood dielectric measurements was $0.0021 \mathrm{nF}$ and $0.00118 \mathrm{nF}$ while the dielectric cell for kidney and liver tissue measurements was $0.016 \mathrm{nF}$ and $0.00912 \mathrm{nF}$ respectively. Booton 7200 impedance meter in conjunction with signal generators (Loadstar, SG416013 and Harris G857993) were used to measure capacitance and dissipation factor of the samples. The dielectric spread parameters $(\alpha)$ of each sample were evaluated from the cole-cole plots.

\section{Results and Analysis}

\subsection{Analysis and Results for Dielectric Spread Parameter $\alpha$, at Low and High Radiofrequency Ranges for Bovine Kidney}

\section{For low Radiofrequency}

1) Linear model:

$$
D=527.0 \alpha-122.0
$$

2) Exponential model:

$$
D=0.0016 \mathrm{e}^{\frac{\alpha_{1}}{0.034}}+0.0016 \mathrm{e}^{\frac{\alpha_{2}}{0.034}}+1.6
$$

3) Polynomial model:

$$
D=11226.0 \alpha^{2}+5683.0 \alpha+712.0
$$

For high Radiofrequency

1) Linear model:

$$
D=264.0 \alpha-25.0
$$

2) Exponential model:

$$
D=14.0 \mathrm{e}^{\frac{-\alpha}{0.184}}+20.1
$$

3) Polynomial model:

$$
D=665.0 \alpha^{2}+31.0 \alpha-0.2
$$

where $D$ and $\alpha$ represents $\gamma$-irradiation dose and spread parameter $\alpha$, respectively. The Akaike Information Criterion (AIC) which is a tool for comparing the performance of two or more model is found to be 56.46, 131.93 and 56.40 for the linear, exponential and polynomial model respectively at low radiofrequency while that of the high radiofrequency was $47.73,56.93$ and 56.03 for linear, exponential and polynomial models respectively. This suggest that Equations 1 and 3 (linear model and polynomial model respectively) are best for low frequency range while and Equation 4 (linear model) is the most appropriate for high radiofrequency response modeling of kidney tissues.

\subsection{Analysis of Bovine Liver Tissue}

For low Radiofrequency

1) Linear model: 


$$
D=363.2 \alpha-42.1
$$

2) Exponential model:

$$
D=-190.4 \mathrm{e}^{\frac{\alpha_{1}}{0.170}}+92.8
$$

3) Polynomial model:

$$
D=-1474.8 \alpha^{2}+941.8 \alpha-93.8
$$

\section{For high Radiofrequency}

1) Linear model:

$$
D=554.5 \alpha-121.3
$$

2) Exponential model:

$$
D=0.202 \mathrm{e}^{\frac{-\alpha}{0.055}}+7.0
$$

3) Polynomial model:

$$
D=6597.7 \alpha^{2}+2964.26 \alpha-338.7
$$

where the $D$ and $\alpha$ represent the $\gamma$-irradiation dose and dielectric spread parameter respectively. The AIC for low radiofrequency models was found to be 60.33 , 69.44 and 69.37 for linear, exponential and polynomial models respectively while that of the high radiofrequency models was 60.71, 69.15 and 68.95 for linear, exponential and polynomial models respectively. Equations (7) and (8) (linear models); and Equation (10) (linear model) are preferred to other models for low and high frequency ranges.

\subsection{Analysis for Bovine Blood Samples}

\section{For low Radiofrequency}

1) Linear model:

$$
D=531.6 \alpha-50.3
$$

2) Exponential model:

$$
D=-269.8 \mathrm{e}^{\frac{\alpha}{0.264}}+126.0
$$

3) Polynomial model:

$$
D=-1060.3 \alpha^{2}+905.4 \alpha-79.3
$$

\section{For high Radiofrequency}

1) Linear model:

$$
D=565.0 \alpha-54.6
$$

2) Exponential model:

$$
D=-267.9 \mathrm{e}^{\frac{-\alpha}{0.507}}+284.9
$$

3) Polynomial model:

$$
D=-1314.0 \alpha^{2}+997.0 \alpha-86.4
$$

where $D$ and $\alpha$ represents the $\gamma$-irradiation dose and dielectric spread parameter 
respectively. The AIC was found to be $40.82,48.15$ and 48.00 respectively for the linear, exponential and polynomial models at low radiofrequency, while the AIC for linear, exponential and polynomial models was 40.80, 48.15 and 48.00 respectively for higher radiofrequency. The AIC of Equation (3) (linear model) and Equation (16) (linear model) suggest that linear model is the preferred model to exponential and polynomial models for bovine blood.

The plot of dielectric spread parameter against gamma $(\gamma)$ irradiation dose shown in Figure 1 revealed that Bovine kidney was more prone to damage (sensitive) at low radiofrequencies followed by the liver tissues and lastly the blood tissue at 0 - $60 \mathrm{~Gy}$; at $85 \mathrm{~Gy}$, the kidney's sensitivity is higher and closely followed by that of blood (see Table 1) while at higher frequency (Figure 2), the sensitivity of liver tissues is higher than that of blood and the kidney tissues

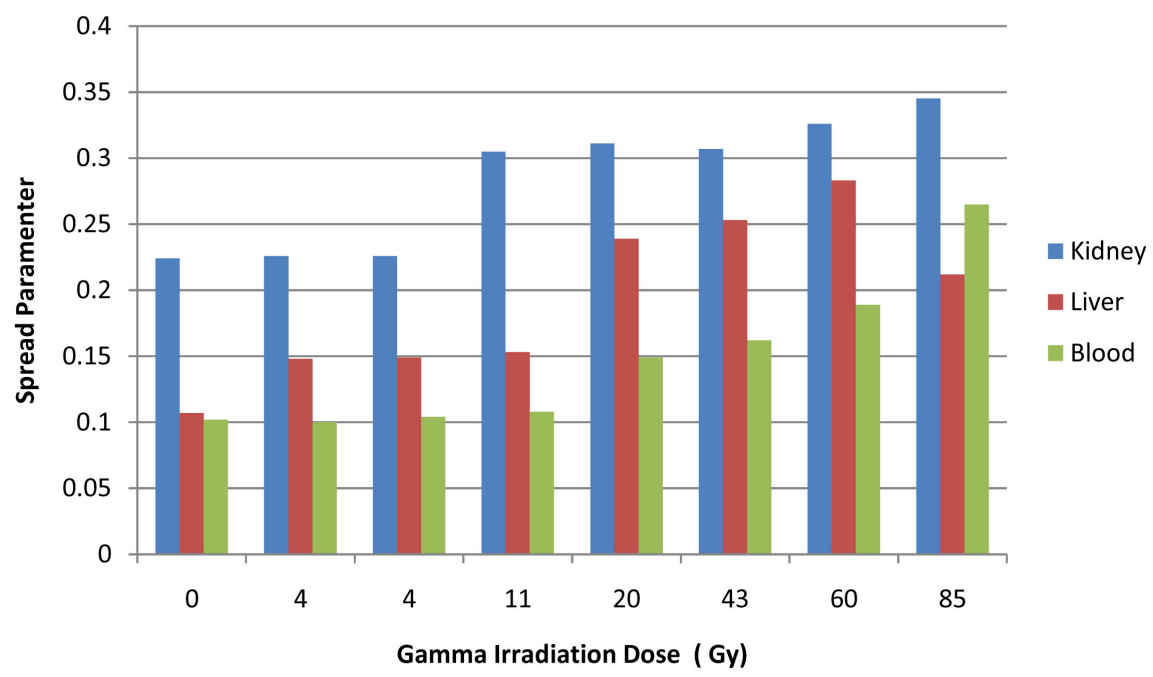

Figure 1. Variation of dielectric spread parameter with Gamma irradiation dose at 0.5 $100.0 \mathrm{kHz}$.

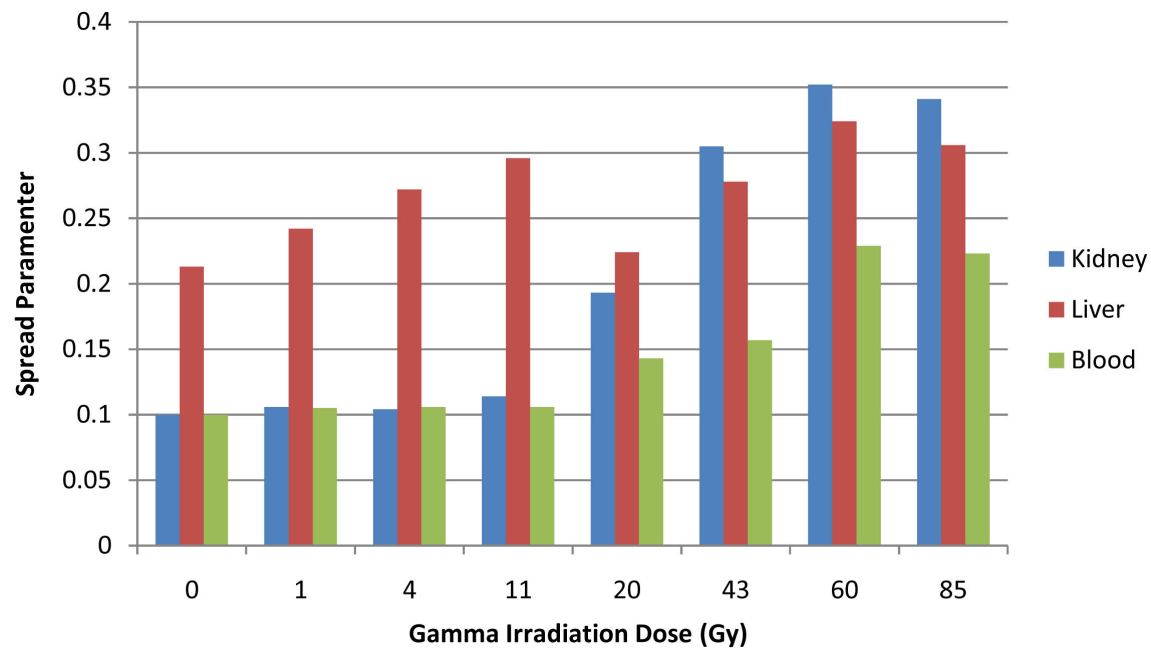

Figure 2. Variation of dielectric spread parameter with Gamma irradiation dose at 0.5 $50.0 \mathrm{MHz}$. 
Table 1. Dielectric spread parameters $\alpha$, of gamma irradiation and non-irradiated bovine kidney, liver and blood at $0.5 \mathrm{kHz}-100.0 \mathrm{kHz}$.

\begin{tabular}{cccc}
\hline \multirow{2}{*}{ Dose (Gy) } & Kidney & Liver & Blood \\
\cline { 2 - 4 } & $\alpha$ & $\alpha$ & $\alpha$ \\
\hline 0 & 0.224 & 0.107 & 0.102 \\
4 & 0.226 & 0.148 & 0.100 \\
4 & 0.226 & 0.149 & 0.104 \\
11 & 0.305 & 0.153 & 0.108 \\
20 & 0.311 & 0.239 & 0.149 \\
43 & 0.307 & 0.253 & 0.162 \\
60 & 0.326 & 0.283 & 0.189 \\
85 & 0.345 & 0.212 & 0.265 \\
\hline
\end{tabular}

especially between the gamma irradiation dose of $0-20 \mathrm{~Gy}$ (see Table 2). The dose range between $45-85$ Gy the sensitivity of kidney was observed to be more than that of the liver tissue and lastly the blood. This result at low frequencies is in good agreement to some extent with the findings of [8] which showed sequential order of the response of gamma irradiated chick embryo as follows; Heart, Kidney, Brain, Lungs, Blood and Liver tissues. The difference in the dielectric response of the bovine kidney, blood and liver tissues at the different frequency ranges may be attributed to the differences in their cellular and tissue morphology resulting in different characteristic polarization of the cell membrane and the relaxation of tissue protein [7] [10] [11]. The difference in water content and ionic conductivity of the three tissues may also account for the difference in dielectric response [12] [13].

The interchange in sensitivity of liver samples and kidney tissue at higher frequencies (Figure 2) may be due to changes in polarization of counter ions near charged surfaces in tissue and possibly, the polarization of the large membrane bound structures and electrolytes [5].

The information derived from this work has application in radiation oncology and dosimetry; mathematical models developed in this work can be used to generate more dielectric data for clinical applications.

\section{Conclusions}

We proposed mathematical response models of gamma irradiated bovine tissues at lower and higher radiofrequencies within gamma irradiation dose regime of 1 - 85 Gy. Using Akaike's Information Criterion, we show that at lower frequencies, linear mathematical models are the most preferred models for describing the response of bovine blood and liver tissues to gamma radiation; both linear and polynomial models are suitable for kidney tissues at lower radiofrequencies while linear models perform better for all the tissues at higher radiofrequencies. 
Table 2. Dielectric spread parameter $\alpha$, of gamma irradiated and non-irradiated bovine kidney, liver and blood at $0.5 \mathrm{MHz}-50.0 \mathrm{MHz}$.

\begin{tabular}{cccc}
\hline \multirow{2}{*}{ Dose $($ Gy $)$} & Kidney & Liver & Blood \\
\cline { 2 - 4 } & $\alpha$ & $\alpha$ & $\alpha$ \\
\hline 0 & 0.100 & 0.213 & 0.100 \\
1 & 0.106 & 0.242 & 0.105 \\
4 & 0.104 & 0.272 & 0.106 \\
11 & 0.114 & 0.296 & 0.106 \\
20 & 0.193 & 0.224 & 0.143 \\
43 & 0.305 & 0.278 & 0.157 \\
60 & 0.352 & 0.324 & 0.229 \\
85 & 0.341 & 0.306 & 0.223 \\
\hline
\end{tabular}

The plot of spread parameters versus gamma irradiation doses shows that at lower radiofrequencies $(0-100 \mathrm{kHz})$ kidney is more sensitive to gamma radiation at dose regime considered, followed by liver tissue except at $85 \mathrm{~Gy}$. While at higher radiofrequencies ( 0 - $50 \mathrm{MHz}$ ), liver between 0 - $20 \mathrm{~Gy}$, while between 43 and 85 Gy the kidney displayed higher sensitivity followed by liver tissues and lastly the blood.

\section{Acknowledgements}

The authors of this work are grateful to Nigeria Nuclear Technology Centre (NNTC) Abuja and the Department of Physics, Benue State University Makurdifor provision of gamma irradiator and laboratory equipments used in this research. We are also grateful to Dr. Agada P.O. of Statistics Department, Federal Universiy of Agriculture Makurdifor his assistance in the analysis of our experimental data.

\section{References}

[1] Hada, M. and Georgakilas, A.G. (2008) Formation of Clustered DNA Damage after High-LET Irradiation: A Review. Journal of Radiation Research, 49, 203-210. https://doi.org/10.1269/jrr.07123

[2] Janonov-Stankov, O., Demajo, M., Djuji, I. and Mandic, M. (2003) Effects of Gamma Irradiation on Magnesium Content in Rat Tissue.

[3] Maliev, V., Popov, D., Jones, J.A. and Casey, R.C. (2007) Mechanism of Action for Anti-Radiation Vaccine in Reducing the Biological Impact of High-Dose Gamma Irradiation. Advances in Space Research, 40, 586-590.

https://www.sciencedirect.com/ https://doi.org/10.1016/j.asr.2007.03.098

[4] Peyman, A. (1996) Dielectric Properties of Tissues as a function of Age and Their Relevance in Assessment of the Exposure of Children to Electromagnetic Field; State of Knowledge. Centre for Radiation Chemical and Environmental Hazards, Health Protection Agency.

[5] Foster, K.R. (2000) Dielectric Properties of Tissues. University of Pennsylvania, 
CRC Press, Boca Raton.

[6] Laogun, A.A., Ajayi, N.O. and Agba, E.H. (2005) Influence of X-Rays on the Radiofrequency Dielectric Properties of Bovine Kidney Tissues. Nigerian Journal of Physics, 17, 117-122.

[7] Agba, E.H., Laogun, A.A. and Ajayi, N.O. (2008) A Comparison of the Effect of Diagnostic X-Rays on the Radiofrequency Dielectric Properties of Bovine Liver with Bovine Kidney Tissues. Nigerian Journal of Physics, 20, 11-22.

[8] Mallidi, S.M., Bhilwade, H.N., Khan, M.Z. and Chamby, R.C. (2007) Gamma Ray Induced Genetic Changes in Different Organs of Chick Embryo Using Peripheral Blood Micronucleus Test and Comet Assay. Mutation Research/Genetic Toxicology and Environmental Mutagenesis, 630, 20-27. http://www.sciencedirect.com https://doi.org/10.1016/j.mrgentox.2007.02.002

[9] Suciu, D. (2005) Model for Explanation of the Difference in Radio-Sensitivity between Dividing and Resting Mammalian Non-Lymphoid Tissue Cells. Heidelberg Journal of Radiation and Environmental Biophysics, 29, 203-211.

[10] Dissado, L.A. (1990) A Fractal Interpretation of the Dielectric Response of Animal Tissues. Physics in Medicine \& Biology, 35, 1487-1503. https://doi.org/10.1088/0031-9155/35/11/005

[11] Stoy, R.D., Foster, K.R. and Schwan, H.P. (1982) Dielectric Properties of Mammalian Tissues from 0.1 to $100.0 \mathrm{MHz}$; a Summary of Recent Data. Physics in Medicine \& Biology, 27, 501-513.https://doi.org/10.1088/0031-9155/27/4/002

[12] Stuchly, M.A., Athley, T.W., Samaras, G.M. and Taylor, G.E. (1982) Measurement of Radiofrequency Permittivity of Biological Tissues with an Open-Ended Coaxial line: Part II-Experimental Results. IEEE Transactions on Microwave Theory and Techniques, 30, 87-91.https://doi.org/10.1109/TMTT.1982.1131022

[13] Schwan, H.P. and Foster, K.R. (1980) RF Field Interaction with Biological Systems: Electrical Properties and Biophysical Mechanisms. Proceedings of the IEEE, 68, 104-113. https://doi.org/10.1109/PROC.1980.11589 